\title{
Effect of Losartan and Atenolol on Heart Rate Variability in Newly Diagnosed Essential Hypertensive Patient.
}

\author{
Shamima Sultana ${ }^{1}$, Shelina Begum ${ }^{2}$, Sultana Ferdousi ${ }^{3}$. \\ ${ }^{1}$ Medical Officer, ${ }^{2}$ Chairman, ${ }^{3}$ Associate Professor, Department of Physiology, BSMMU.
}

\begin{abstract}
:
Background: Essential hypertension is associated with altered autonomic function ${ }^{1}$. Essential hypertension is treated with drugs which modify the sympatho- parasympathetic balance. Losartan (angiotensin II receptor blocker) and atenolol (beta blocker) is commonly used antihypertensive drugs. Objective: To evaluate the effect of antihypertensive drugs on heart rate variability (HRV) in patients with essential hypertension. Methods: This prospective observational study was carried out in the Department of Physiology, Bangabandhu Sheikh Mujib Medical University (BSMMU), Shahbag, Dhaka from July 2012 to June 2013 on 120 newly diagnosed hypertensive patients without any medication (group B, age 30-55 years). They were selected from the Out Patients Department (OPD) of cardiology, BSMMU, Dhaka. Age, sex and BMI matched 60 apparently healthy normotensive subjects were also studied as control (group A). Based on treatment, these study subjects were divided into two groups (B1 and B2). GroupB1 included 60 patients received losartan $50 \mathrm{mg}$ daily and $\mathrm{B}_{2 \mathrm{a}}$ included 60 patients received atenolol $50 \mathrm{mg}$ daily. They were observed once before the treatment $\left(\mathrm{B}_{1 \mathrm{a}} \& \mathrm{~B}_{2 \mathrm{a}}\right)$, after 3 months medication $\left(B 1_{b} \& B 2_{b}\right)$ and after 6 months medication $\left(B 1_{c} \& B 2_{c}\right)$. For assessing HRV, Mean heart rate, Mean R-R interval, Max/Min R-R interval, SDNN, RMSSD were recorded by a polyrite. Data were compared among before treatment, after 3 months treatment and after 6 months treatment. For statistical analysis ANOVA, independent sample ' $t$ ' test and paired sample ' $t$ ' test were performed. Results: Mean resting pulse rate, mean heart rate, systolic blood pressure, diastolic blood pressure were significantly higher and mean R-R interval, Max/Min R-R interval, SDNN, RMSSD were significantly lower in newly diagnosed hypertensive patients in comparison with that of healthy normotensive subjects and after treatment. In both groups SDNN, RMSSD, mean R-R interval were found significantly higher after 6 months of treatment compared to their values after 3 months treatment. Again these values were found close to the values in normotensive subjects. In addition, mean heart rate was found significantly lower in atenolol treated patients than those of controls. Again in atenolol group these values were found significantly higher than the corresponding values in losartan treated patients after 6 months treatment. Conclusion: Reduced cardiac vagal tone occurs in newly diagnosed hypertensive patients which is improved by both losartan and atenolol and in particular atenolol was found more effective.
\end{abstract}

Key words: Newly diagnosed hypertensive patients, heart rate variability.

[BSMMU J 2013; 6 (2) : 161-167]

\section{Introduction:}

Hypertension has been recognized as the commonest cardiovascular problems in most of the advanced countries and developing countries. Essential hypertension accounts for $90-95 \%$ of all cases of hypertension. Hypertension is an emerging health problem in Bangladesh

Address for Correspondence: Dr. Shamima Sultana Medical Officer, Department of Physiology, BSMMU. Mobile: 01711177678 among the cardiovascular diseases. The prevalence rate of hypertension is very high and more than $20 \%$ of the adults have hypertension ${ }^{2}$.

The autonomic nervous system (ANS)) plays a fundamental role in the control of arterial blood pressure and heart rate. Essential hypertension is associated with altered autonomic function ${ }^{1}$. Hypertension is characterized by sympathetic overactivity ${ }^{3}$ and attenuation of parasympathetic modulation of the heart ${ }^{4}$. 
The heart rate variability is a powerful noninvasive tool in the assessment of the cardiac autonomic nerve function ${ }^{5}$. Several studies have identified reduced HRV in essential hypertension ${ }^{3,6,7,8}$. Reduced HRV has been correlated with increased mortality after acute myocardial infraction ${ }^{9,10}$.

Among the various HRV measures mean R-R interval, mean heart rate, maximum and minimum $\mathrm{R}-\mathrm{R}$ ratio, SDNN and RMSSD usually used as marker for cardiac vagal activity ${ }^{11}$. Essential hypertension is treated with various antihypertensive drugs which modify the sympatho- parasympathetic balance ${ }^{3,12}$. A prospective randomized trial revealed that losartan significantly increased parasympathetic activity in hypertensiye patients when compared to placebo $^{13}$. Few studies reported losartan inhibit sympathetic activity in hypertensive patients ${ }^{14,15}$. Another study revealed that losartan had no significant effect on HRV in hypertensive patients ${ }^{16,17}$. Few studies revealed that autonomic balance shifted toward the increased vagal activity treated with atenolol in hypertensive patients ${ }^{17,18}$. On the other hand, one study reported that atenolol had no significant effect on $\mathrm{HRV}^{23}$.

Although several investigators observed the effect of losartan and atenolol on HRV separately but no report compared the effect of losartan and atenolol on HRV in newly diagnosed hypertensive patients. Therefore this study aimed to evaluate HRV in untreated hypertensive patients and compare the effect of losartan and atenolol on HRV.

\section{Methods:}

This prospective observational study was carried out to observe the HRV by assessing time domain measures in 120 newly diagnosed hypertensive patients with ranged from 30-55 years in the Department of Physiology, Bangabandhu Sheikh Mujib Medical University (BSMMU), Shahbag, Dhaka from July 2012 to June 2013. Age, sex and BMI matched 60 apparently healthy normotensive subjects were also studied as control (group A). Based on treatment, these study subjects were divided into two groups(B1 and B2). GroupB1 ${ }_{a}$ included 60 patients received losartan $50 \mathrm{mg}$ daily and $\mathrm{B} 2_{\mathrm{a}}$ included 60 patients received atenolol $50 \mathrm{mg}$ daily. They were observed once before the treatment $\left(B 1_{\mathrm{a}} \& \mathrm{~B} 2_{\mathrm{a}}\right)$ after3 months medication $\left(\mathrm{B} 1_{\mathrm{b}} \& \mathrm{~B} 2_{\mathrm{b}}\right)$ and after 6 months medication ( $\left.\mathrm{B} 1_{\mathrm{c}} \& \mathrm{~B} 2_{\mathrm{c}}\right)$. These patients were selected from the Out Patient Department of Cardiology, BSMMU.

Then the subject was prepared for Autonomic Nerve Function Test. The subject was kept in complete bed rest in supine position for 15-20 minutes in a cool and calm environment. Then all preparations for recording of the Heart rate variability parameters were made by connecting the channels of ECG and a 5 minutes recording was taken in resting position. Time domain parameters of the HRV like Mean heart rate, Mean R-R interval, Max/Min R-R interval, SDNN, RMSSD were measured by polygraph.

Then all the patients were requested to attend the Department of Physiology of BSMMU, again after 3 months and after 6 months of antihypertensive medication, to have the assessment of the above mentioned study variables. For statistical analysis ANOVA, independent sample ' $t$ ' test and paired sample ' $t$ ' test were performed.

\section{Results:}

\section{Table-I}

Anthopometric data of all subjects are given in.

\begin{tabular}{lcc}
\hline Group & Age (years) & BMI $\left(\mathrm{kg} / \mathrm{m}^{2}\right)$ \\
\hline $\mathrm{A}(\mathrm{n}=60)$ & $42.04 \pm 1.134$ & $23.24 \pm 0.243$ \\
& $(30-35)$ & $(18.59-24.77)$ \\
$\mathrm{B}_{1 \mathrm{a}}(\mathrm{n}=60)$ & $41.64 \pm 1.165$ & $23.27 \pm 0.216$ \\
& $(30-35)$ & $(18.65-24.77)$ \\
$\mathrm{B}_{2 \mathrm{a}}(\mathrm{n}=60)$ & $44.74 \pm 1.321$ & $23.01 \pm 0.177$ \\
& $(30-35)$ & $(18.37-24.68)$
\end{tabular}

Statistical analysis

\begin{tabular}{lll}
\hline Group & \multicolumn{2}{c}{ P value } \\
\hline $\mathrm{A} v s ~_{1 \mathrm{a}}$ vs B $_{2 \mathrm{a}}^{\xi}$ & $0.296^{\mathrm{ns}}$ & $0.850^{\mathrm{ns}}$ \\
$\mathrm{A} v s ~ B_{1 \mathrm{a}} \varphi$ & $0.806^{\mathrm{ns}}$ & $0.912^{\mathrm{ns}}$ \\
$\mathrm{A} v s ~_{2 \mathrm{a}} \varphi$ & $0.124^{\mathrm{ns}}$ & $0.656^{\mathrm{ns}}$ \\
$\mathrm{B}_{1 \mathrm{a}}$ vs B $_{2 \mathrm{a}}{ }^{\mathrm{ns}}$ & $0.533^{\mathrm{ns}}$ & $0.748^{\mathrm{ns}}$ \\
\hline
\end{tabular}

Data were expressed as mean $\pm \mathrm{SE}$, figures in parenthesis indicate ranges. $\xi=$ one way anova. $\varphi=$ independent sample 't' test. $\Omega=$ paired sample 't' test. 


$$
\begin{aligned}
& \text { Group } \mathrm{A}=\text { Apparently healthy subject (control) } \\
& \mathrm{B}_{1}=\text { Newly diagnosed hypertensive patients } \\
& \text { before treatment } \\
& \mathrm{B}_{2}=\text { After } 3 \text { months treatment with losartan } \\
& \mathrm{B}_{3}=\text { After } 3 \text { months treatment with losartan } \\
& * * *=\mathrm{p}<0.001 \quad \mathrm{~ns}=\text { non significant }(\mathrm{p}>0.05) \\
& * *=\mathrm{p}<0.01 \quad \mathrm{n}=\text { number of subjects. } \\
& * \quad=\mathrm{p}<0.05
\end{aligned}
$$

Mean resting pulse, systolic blood pressure and diastolic

\begin{tabular}{|c|c|c|c|}
\hline Group & & P valu & \\
\hline $\begin{array}{l}\text { A vs } B_{1 a} \\
\text { vs } B_{2 a} \\
\text { vs } B_{3 a}{ }^{\xi}\end{array}$ & $0.000^{* * *}$ & $0.000^{* * *}$ & $0.000 * * *$ \\
\hline A vs $B_{1 a}{ }^{\varphi}$ & $0.000^{* * *}$ & $0.000 * * *$ & $0.000^{* * *}$ \\
\hline$A$ vs $B_{2 a}{ }^{\varphi}$ & $0.000 * * *$ & $0.000 * * *$ & $0.000^{* * *}$ \\
\hline
\end{tabular}
blood pressure were significantly higher in group $\mathrm{B}_{1 \mathrm{a}}$ and

\begin{tabular}{|c|c|c|c|}
\hline $\mathrm{B}_{1 \mathrm{a}}$ vs $\mathrm{B}_{\mathrm{lb}}{ }^{\Omega}$ & $0.000^{* * *}$ & $0.000^{* * *}$ & $0.000 * * *$ \\
\hline $\mathrm{B}_{1 \mathrm{~b}}$ vs $\mathrm{B}_{1 \mathrm{c}}{ }^{\Omega}$ & $0.252 \mathrm{~ns}$ & $0.095 \mathrm{~ns}$ & $0.047^{*}$ \\
\hline $\mathrm{B}_{\mathrm{la}} \mathrm{vs} \mathrm{B}_{1 \mathrm{c}}^{\Omega}$ & $0.000^{* * *}$ & $0.000^{* * *}$ & $0.000^{* * *}$ \\
\hline $\mathrm{B}_{2 \mathrm{a}}$ vs $\mathrm{B}_{2 \mathrm{~b}}{ }^{\Omega}$ & $0.000^{* * *}$ & $0.000 * * *$ & $0.000 * * *$ \\
\hline $\mathrm{B}_{2 \mathrm{~b}}$ vs $\mathrm{B}_{2 \mathrm{c}}{ }^{\Omega}$ & $0.000^{* * *}$ & $0.038^{*}$ & $0.006^{* *}$ \\
\hline $\mathrm{B}_{2 \mathrm{a}}$ vs $\mathrm{B}_{2 \mathrm{c}}{ }^{\Omega}$ & $0.000^{* * *}$ & $0.000 * * *$ & $0.000 * * *$ \\
\hline
\end{tabular}
$\mathrm{B}_{2 \mathrm{a}}$ and this values were significantly decreased in group $\mathrm{B}_{1 \mathrm{~b}}, \mathrm{~B}_{1 \mathrm{c}}, \mathrm{B}_{2 \mathrm{~b}}$ and $\mathrm{B}_{2 \mathrm{c}}$.(TableII)

\section{Table-II}

Baseline measures in different groups $(n=120)$

\begin{tabular}{lccc}
\hline Groups & Pulse & $\begin{array}{c}\text { systolic } \\
\text { blood } \\
\text { pressure }\end{array}$ & $\begin{array}{c}\text { Diastolic } \\
\text { blood } \\
\text { pressure }\end{array}$ \\
\hline $\mathrm{A}$ & $78.15 \pm 0.862$ & $126.4 \pm 0.603$ & $73.2 \pm 0.515$ \\
& $(93-70)$ & $(120-135)$ & $(65-80)$ \\
$\mathrm{B}_{1 \mathrm{a}}$ & $83.55 \pm 0.919$ & $141.6 \pm 0.462$ & $91.5 \pm 0.295$ \\
& $(95-70)$ & $(140-155)$ & \\
$\mathrm{B}_{1 \mathrm{~b}}$ & $76.37 \pm 0.767$ & $127.7 \pm 0.45$ & $77.4 \pm 0.510$ \\
& $(90-70)$ & $(120-135)$ & $(70-85)$ \\
$\mathrm{B}_{\mathrm{IC}}$ & $75.33 \pm 0.664$ & $127.7 \pm 0.449$ & $76.2 \pm 0.476$ \\
& $(87-65)$ & $(120-135)$ & $(70-85)$ \\
$\mathrm{B}_{2 \mathrm{a}}$ & $94.63 \pm 0.636$ & $147.6 \pm 0.561$ & $92.5 \pm 0.333$ \\
& $(106-87)$ & $(140-155)$ & $(90-98)$ \\
$\mathrm{B}_{2 \mathrm{~b}}$ & $70.80 \pm 0.517$ & $126.3 \pm 0.473$ & $75.9 \pm 0.468$ \\
& $(77-62)$ & $(120-130)$ & $(70-80)$ \\
$\mathrm{B}_{2 \mathrm{c}}$ & $65.17 \pm 0.495$ & $125.5 \pm 0.449$ & $74.6 \pm 0.502$ \\
& $(68-52)$ & $(120-135)$ & $(70-80)$ \\
\hline
\end{tabular}

Statistical analysis
Data were expressed as mean $\pm \mathrm{SE}$, figures in parenthesis indicate ranges. $\xi=$ one way anova. $\varphi=$ independent sample 't' test. $\Omega=$ paired sample ' $t$ ' test.

$\mathrm{SBP}=$ Systolic blood pressure .

DBP $=$ Diastolic blood pressure

Group $\mathrm{A}=$ Apparently healthy subject (control)

$\mathrm{B}_{1 \mathrm{a}}=$ Newly diagnosed hypertensive patients before treatment

$\mathrm{B}_{\mathrm{Ib}}=$ After 3 months treatment with losartan

$\mathrm{B}_{1 \mathrm{c}}=$ After 6 months treatment with losartan

$\mathrm{B}_{2 \mathrm{a}}=$ Newly diagnosed hypertensive patients before treatment

$\mathrm{B}_{2 \mathrm{~b}}=$ After 3 months treatment with atenolol

$\mathrm{B}_{2 \mathrm{c}}=$ After 6 months treatment with atenolol

$$
\begin{array}{lll}
* * * & =\mathrm{p}<0.001 & \mathrm{~ns}=\text { non significant }(\mathrm{p}>0.05) \\
* * & =\mathrm{p}<0.01 & \mathrm{n}=\text { number of subjects. } \\
* & & =\mathrm{p}<0.05
\end{array}
$$

Mean values of mean heart rate were significantly higher in group $\mathrm{B}_{1 \mathrm{a}}$ and $\mathrm{B}_{2 \mathrm{a}}$ than that of group $\mathrm{A}$ and this values were significantly decreased in group $\mathrm{B}_{\mathrm{b}}$ and group $\mathrm{B}_{1 \mathrm{c}}$ in comparison to their baseline value. In addition this value showed no significant difference when compared between group $A$ and group $B_{1 c}$. Mean heart rate in group $B_{2 b}$ and group $B_{2 c}$ was significantly lower compared to their baseline value. Mean heart rate in group $\mathrm{B}_{\mathrm{lc}}$ was significantly lower than that of control group. Mean R-R interval, Max/Min R-R interval were significantly lower in group $\mathrm{B}_{1 \mathrm{a}}$ and $\mathrm{B}_{2 \mathrm{a}}$ than that of group $\mathrm{A}$ and this values were significantly increased in group $B_{1 b}, B_{1 c}, B_{2 b}$ and $B_{2 c}$ in compared to baseline value. (Table III). 
Table-III

Simple time domain measures of HRV in different groups

\begin{tabular}{lccc}
\hline Groups & $\begin{array}{c}\text { Mean heart } \\
\text { rate(Beat/ } \\
\text { min })\end{array}$ & $\begin{array}{c}\text { Mean R-R } \\
\text { interval } \\
\text { (sec.) }\end{array}$ & $\begin{array}{c}\text { Max/min R-R } \\
\text { interval }\end{array}$ \\
\hline $\mathrm{A}(\mathrm{n}=60)$ & $73.33 \pm 0.712$ & $0.753 \pm 0.12$ & $1.75 \pm 0.066$ \\
& $(85-65)$ & $(0.91-0.6)$ & $(3.17-1.19)$ \\
$\mathrm{B}_{1 \mathrm{a}}(\mathrm{n}=60)$ & $84.85 \pm 0.895$ & $0.693 \pm 0.014$ & $1.46 \pm 0.041$ \\
& $(95-70)$ & $(1.04-0.571)$ & $(3.79-1.12)$ \\
$\mathrm{B}_{1 \mathrm{~b}}$ & $77.75 \pm 0.909$ & $0.730 \pm 0.10$ & $1.71 \pm 0.64$ \\
& $(90-65)$ & $(1.128-0.619)$ & $(4.99-1.104)$ \\
$\mathrm{B}_{1 \mathrm{c}}$ & $74 \pm 0.886$ & $0.748 \pm 0.011$ & $1.78 \pm 0.057$ \\
& $(85-63)$ & $(1.135-.551)$ & $(12.18-.789)$ \\
$\mathrm{B}_{2 \mathrm{a}}$ & $97.33 \pm 0.864$ & $0.624 \pm 0.007$ & $1.41 \pm 0.055$ \\
& $(113-87)$ & $(.80-.531)$ & $(2.79-1.06)$ \\
$\mathrm{B}_{2 \mathrm{~b}}$ & $73.82 \pm 0.543$ & $0.760 \pm 0.012$ & $1.81 \pm 0.070$ \\
& $(84-65)$ & $(2.39-.562)$ & $(4.26-1.16)$ \\
$\mathrm{B}_{2 \mathrm{c}}$ & $61.8 \pm 0.556$ & $0.923 \pm 0.019$ & $1.93 \pm 0.071$ \\
& $(69-52)$ & $(2.34-0.658)$ & $(2.88-1.12)$ \\
\hline & & &
\end{tabular}

Statistical analysis

\begin{tabular}{|c|c|c|c|}
\hline Group & PValue & & \\
\hline$A$ vs $B_{1 a}^{\varphi}$ & $0.000^{* * *}$ & $0.001 * * *$ & $0.000 * * *$ \\
\hline$A$ vs $B_{1 b}{ }^{\circ}$ & 0.000 & $0.122 \mathrm{~ns}$ & $0.714 \mathrm{~ns}$ \\
\hline$A$ vs $B_{1 c}^{\varphi}$ & $0.243 \mathrm{~ns}$ & $0.747 \mathrm{~ns}$ & $0.694 \mathrm{~ns}$ \\
\hline $\mathrm{B}_{1 \mathrm{a}} \mathrm{vs} \mathrm{B}_{1 \mathrm{~b}}^{\Omega}$ & 0.000 & $0.024^{*}$ & $0.002 * *$ \\
\hline $\mathrm{B}_{1 \mathrm{~b}}$ vs $\mathrm{B}_{1 \mathrm{c}}^{\Omega}$ & 0.008 & 0.239 & $0.411 \mathrm{~ns}$ \\
\hline $\mathrm{B}_{1 \mathrm{a}}$ vs $\mathrm{B}_{1 \mathrm{c}}^{\Omega}$ & $0.000 * * *$ & $0.001^{* * *}$ & $0.000 * * *$ \\
\hline$A$ vs $B_{2 a^{\circ}}$ & $0.000 * * *$ & $0.001^{* * *}$ & $0.000^{* * *}$ \\
\hline$A$ vs $B_{2 b}{ }^{\varphi}$ & $0.591 \mathrm{~ns}$ & $0.651 \mathrm{~ns}$ & $0.481 \mathrm{~ns}$ \\
\hline $\mathrm{A}$ vs $\mathrm{B}_{2 \mathrm{c}}{ }^{\varphi}$ & $0.000 * * *$ & $0.001^{* * *}$ & $0.056 \mathrm{~ns}$ \\
\hline $\mathrm{B}_{2 \mathrm{a}}$ vs $\mathrm{B}_{2 \mathrm{~b}}^{\Omega}$ & $0.000^{* * *}$ & $0.001^{* * *}$ & $0.000^{* * *}$ \\
\hline $\mathrm{B}_{2 \mathrm{~b}} \mathrm{vs} \mathrm{B}_{2 \mathrm{c}}^{\Omega}$ & $0.000^{* * *}$ & $0.001^{* * * *}$ & $0.190 \mathrm{~ns}$ \\
\hline $\mathrm{B}_{2 \mathrm{a}} \mathrm{vs} \mathrm{B}_{2 \mathrm{c}}^{\Omega}$ & $0.000^{* * *}$ & $0.001^{* * *}$ & $0.000 * * *$ \\
\hline
\end{tabular}

Data were expressed as mean $\pm \mathrm{SE}$, figures in parenthesis indicate ranges. $\varphi=$ independent sample ' $t$ ' test. $\Omega$ =paired sample ' $t$ ' test.

$\mathrm{R}-\mathrm{R}$ = interval between successive $\mathrm{QRS}$ complex(sec)

Max $=$ maximum
Min $=$ minimum

Group A = Apparently healthy subject (control)

$\mathrm{B}_{1 \mathrm{a}} \quad=$ Newly diagnosed hypertensive patients before treatment

$\mathrm{B}_{1 \mathrm{~b}}=$ After 3 months treatment with losartan

$\mathrm{B}_{1 \mathrm{c}}=$ After 6 months treatment with losartan

$\mathrm{B}_{2 \mathrm{a}} \quad=$ Newly diagnosed hypertensive patients before treatment

$\mathrm{B}_{2 \mathrm{~b}}=$ After 3 months treatment with atenolol

$\mathrm{B}_{2 \mathrm{c}}=$ After 6 months treatment with atenolol

$* * * \quad=p<0.001 \quad n s=$ non significant $(p>0.05)$

** $\quad=p<0.01 \quad n=$ number of subjects.

* $\quad=\mathrm{p}<0.05$

The mean values of SDNN, RMSSD were significantly lower in group $\mathrm{B}_{1 \mathrm{a}}$ and $\mathrm{B}_{2 \mathrm{a}}$ than that of group $\mathrm{A}$ and this values were significantly increased in group $B_{1 b}, B_{1 c}, B_{2 b}$ and $B_{2 c}$ than that of their baseline. But these values showed no statistically significant difference between group A and group B1c. (Table IV).

\section{Table-IV}

Statistical time domain measures of HRV in different groups $(n=120)$

\begin{tabular}{lcc}
\hline Groups & SDNN $(\mathrm{ms})$ & RMSSD $(\mathrm{ms})$ \\
\hline $\mathrm{A}(\mathrm{n}=60)$ & $78.18 \pm 1.95$ & $32.69 \pm 0.89$ \\
& $(99.8-50.09)$ & $(41.89-17.99)$ \\
$\mathrm{B}_{1 \mathrm{a}}(\mathrm{n}=60)$ & $40.63 \pm 2.37$ & $24.57 \pm 0.521$ \\
& $(102.8-15.11)$ & $(32.99-18.76)$ \\
$\mathrm{B}_{1 \mathrm{~b}}$ & $54.40 \pm 2.39$ & $26.98 \pm 0.392$ \\
& $(100.09-26.15)$ & $(34 .-22)$ \\
$\mathrm{B}_{1 \mathrm{c}}$ & $71.42 \pm 2.53$ & $30.59 \pm .414$ \\
& $(108.1-31.09)$ & $(45.31-23.8)$ \\
$\mathrm{B}_{2 \mathrm{a}}$ & $27.05 \pm 1.94$ & $22.43 \pm .194$ \\
& $(81-10.98)$ & $(25.6-19.9)$ \\
$\mathrm{B}_{2 \mathrm{~b}}$ & $51.3 \pm 2.57$ & $24.86 \pm .291$ \\
& $(108-18.5)$ & $(28.7-21.3)$ \\
$\mathrm{B}_{2 \mathrm{c}}$ & $81.68 \pm 3.67$ & $31.21 \pm .371$ \\
\hline
\end{tabular}


Statistical analysis

\begin{tabular}{|c|c|c|}
\hline \multirow{2}{*}{$\frac{\text { Group }}{\mathrm{Avs}^{\mathrm{B}_{1}{ }^{\varphi}}}$} & \multicolumn{2}{|c|}{$P$ value } \\
\hline & $0.000 * * *$ & $0.000^{* * *}$ \\
\hline A vs $B_{2}{ }^{\varphi}$ & $0.000 * * *$ & $0.000 * * *$ \\
\hline$A$ vs $B_{3}^{\varphi}$ & 0.036 & 0.035 \\
\hline $\mathrm{B}_{1 \mathrm{a}}$ vs $\mathrm{B}_{1 \mathrm{~b}}{ }^{\Omega}$ & $0.000 * * *$ & 0.001 \\
\hline $\mathrm{B}_{1 \mathrm{~b}}$ vs $\mathrm{B}_{1 \mathrm{c}}^{\Omega}$ & $0.000 * * *$ & 0.008 \\
\hline $\mathrm{B}_{1 \mathrm{a}}$ vs $\mathrm{B}_{1 \mathrm{c}} \Omega$ & $0.000 * * *$ & $0.000 * * *$ \\
\hline$A$ vs $B_{2 a}{ }^{\phi}$ & $0.000 * * *$ & $0.000 * * *$ \\
\hline$A$ vs $B_{2 b}{ }^{\varphi}$ & $0.000 * * *$ & $0.000 * * *$ \\
\hline$A$ vs $B_{2 c}{ }^{\varphi}$ & $0.40 \mathrm{Ins}$ & $0.000^{* * * *}$ \\
\hline $\mathrm{B}_{2 \mathrm{a}}$ vs $\mathrm{B}_{2 \mathrm{~b}}^{\Omega}$ & $0.000 * * *$ & $0.000^{* * *}$ \\
\hline $\mathrm{B}_{2 \mathrm{~b}}$ vs $\mathrm{B}_{2 \mathrm{c}} \Omega$ & $0.000^{* * *}$ & $0.000^{* * * *}$ \\
\hline $\mathrm{B}_{2 \mathrm{a}} \mathrm{vs} \mathrm{B} \mathrm{B}_{2 \mathrm{c}}$ & $0.000^{* * *}$ & $0.000 * * *$ \\
\hline
\end{tabular}

Data were expressed as mean $\pm \mathrm{SE}$, figures in parenthesis indicate ranges. $\varphi=$ independent sample 't' test. $\Omega=$ paired sample ' $t$ ' test.

$\mathrm{SDNN}=$ Standard deviation of $\mathrm{N}-\mathrm{N}$ interval

RMSSD $=$ Square root of mean squared differenceso between adjacent $\mathrm{NN}$ intervals.

$$
\begin{aligned}
& \text { Group } \mathrm{A}=\text { Apparently healthy subject (control) } \\
& \mathrm{B}_{1 \mathrm{a}}=\text { Newly diagnosed hypertensive patients } \\
& \text { before treatment } \\
& \mathrm{B}_{1 \mathrm{~b}}=\text { After } 3 \text { months treatment with losartan } \\
& \mathrm{B}_{1 \mathrm{c}}=\text { After } 3 \text { months treatment with losartan } \\
& B_{2 a}=\text { Newly diagnosed hypertensive patients } \\
& \text { before treatment } \\
& \mathrm{B}_{2 \mathrm{~b}}=\text { After } 3 \text { months treatment with atenolol } \\
& \mathrm{B}_{2 \mathrm{c}}=\text { After } 3 \text { months treatment with atenolol } \\
& * * *=\mathrm{p}<0.001 \mathrm{~ns}=\text { non significant }(\mathrm{p}>0.05) \\
& * * \quad=\mathrm{p}<0.01 \mathrm{n}=\text { number of subjects. } \\
& \text { * } \quad=\mathrm{p}<0.05
\end{aligned}
$$

\section{Discussion:}

In the present study, values of the HRV parameters in healthy normotensive group were almost similar to other investigators $^{5,19}$.
In this study, the mean resting pulse rate, mean heart rate, resting systolic and diastolic blood pressure were found significantly higher in newly diagnosed hypertensive patients than that of healthy normotensive groups. These values were found significantly lower after 6 months treatment compared to their values after 3 months treatment. Again these values were found close to the values in normotensive subjects. Similar type of findings were also reported by the various investigators from different countries ${ }^{19,20,24}$. Again the mean heart rate was found significantly lower in atenolol treated patients than those of controls.

Again in this study significantly lower the mean R-R interval , Max/Min R-R interval, SDNN and RMSSD in untreated hypertensive patients indicate lower cardiac vagal modulation .Result of these parameters after 3 months and 6 months treatment with both losartan and atenolol group showed significant improvement. Again when the effect of this two drugs on cardiac autonomic nerve function was compared, the result of the study showed atenolol is more effective than losartan in increasing the cardiac vagal activity supported by the significantly higher value of mean R-R interval, SDNN, RMSSD and lower value of mean heart rate in atenolol treated hypertensive subjects. These values were found significantly higher after 6 months treatment compared to their values after 3 months treatment ${ }^{15,17,21}$. Again these values were found close to the values in normotensive subjects. Moreover this value was significantly higher in atenolol treated group than that of losartan treated group. Similar findings in these two parameters were also reported by the various investigators of different countries $^{1,15,17,18,22,24}$.

Investigators suggested that essential hypertension is associated with altered autonomic regulation, which is supported by reduced HRV, sympathetic over activity and low Baroreflex sensitivity (BRS) $)^{5,17,20,21}$. 
Different investigators suggested various mechanisms for the improvement of HRV in hypertensive patients after treatment with losartan and atenolol. It has been suggested that blocking the type-I receptor $\left(\mathrm{AT}_{1}\right)$ of Angiotensin II by losartan would release the baroreceptor from the suppresor effect of high level of Angiotensin II leading to increase BRS which in turn bring the sympathovagal balance back towards normal. The benefit of blocking the type-I receptor $\left(\mathrm{AT}_{1}\right)$ of Angiotensin II by losartan is also derived from its ability to increase NO production from vascular endothelium and neuron which in turn facilitate the regulation of BRS and HRV in losartan treated hypertensive group ${ }^{25}$. Furthermore, NO has been demonstrated to augment cardiac vagal control in human suggesting beneficial effect on BRS and $H R V^{26}$.

Beta blocker lower blood pressure predominently by inhibiting beta- 1 adrenergic receptors, cardiac contractility and heart rate which in turn facilitates vagal activity. Renin release and angiotensin II are also inhibited by this mechanisms. Additionally, beta blocker alter BRS, and increase prostacyclin biosynthesis, thereby facilitating vasodilatation $^{27}$.

The exact mechanisms involved for the decreased HRV and BRS in hypertension and improvement after treatment with losartan and atenolol in hypertensive groups of present study can not be elucidated from this type of study. However, it is assumed that all the above mentioned mechanisms may influence the degree of deterioration of these variables in hypertension patients and improvement after treatment with losatan and atenolol. From the result though the improvement of cardiac autonomic nerve function was marked after 3 months of treatmebt but data after 6 months of treatment showed further improvement. This is suggestive of positive relation and duration of treatment with antihypertenstve drugs with improvement of HRV.

\section{Conclusion:}

Impairment of cardiac autonomic nervous activity occurs in newly diagnosed hypertensive patients which is improved by both losartan and atenolol and in particular atenolol was found more effective. The rate of improvement of impaired cardiac autonomic function releated to their duration of treatment.

\section{References:}

1. Sahin I, Kosar F, Altunkan S, Gunaydin M.(2004) Comparision of the effects of amlodipine and verapamil on autonomic activity in hypertensive patients. Eur J of internal med. 15: 225-230.

2. Sahriar I, Haque J, Momen A.(2004) Paterns of antihypertensive drugs used in two postgraduate institutions. Journal of Dhaka National medical College \& Hospital. 13(1): 41-44.

3. Guzzetti S, Piccaluga E, Casati R, et al. (1988) Sympathetic predominance in essential hypertension: A study employing spectral analysis of heart rate variability. J Hypertens. 6: 711-717.

4. Langewitz W, Ruddel H, Schachinger, H.F. 1994. Reduced parasympathetic cardiac control in patients with hypertension at rest and under mental stress. Am. Heart J. 1994; 127:122-8.

5. Singh JP, Larson MG, Tsuji H, et al.(1998) Reduced heart rate variability in new onset hypertension. Insights into pathogenesis of hypertension: The Framingham heart study. Hypertension. 32:293-297.

6. Chakko S, Mulingtapang RF, Huikuri HV, Kessler KM, Materson BJ, Myeberg RJ. (1993) Alteration in heart rate variability and its circadian rhythm in hypertensive patients with left ventricular hypertrophy free of coronary artery disease. Am. Heart J. 126 : 1364-1372.

7. Takalo R, Korhonen I, Turjanamaa V, Majahalme S, Tuomisto M, Uusitalo A.(1994) Short term variability of blood pressure and heart rate in borderline and mild hypersensitive subjects. Hypertension. 23:18-24.

8. Huikuri HV, Ylitalo A, Pikkujamasa SM, et al.(1996) Heart rate variability in systemic hypertension. Am J Cardiol . 77:10731077.

9. Kleiger RE, Miller JP, Bigger JT Jr, et al. (1987) Decreased heart rate variability and its association with increased mortality after acute myocardial infarction. Am J cardiol. $59: 256-262$.

10. Bigger JT, Fleiss JL, SteinmannRC, Rolnitzk LM, Kleiger RE, Rottman JN.(1992) Frequency domain measures of heart rate variability and mortality after myocardial infarctions. Circulation. 85: $164-71$

11. Task Force Of The European Society Of Cardiology And The North American Society Of Pacing And Electrophysiology, Heart Rate Variability. Standards Of Measurement, Physiological Interpretation And Clinical Use. (1996) Circulation. 93: 1043- 65.

12. Pagani M, Lucini D. (2001) Autonomic dysregulation in essential hypertension: Insight from heart rate and arterial pressure variability. Auton. Neurosci. 90:76-82. 
13. Krum H, Lambert E, Windelbank E, Campbell DJ, Esler M. ( 2006) Effect of angiotensin receptor blockade on autonomic nervous system function in patients with essential hypertension. Am J Physiol Heart Circ Physiol. 290 : H1706-HI712.

14. Ozdemir M, Arslan U, Turkoglu S, balecbgut S, Cengel A. (2008) Losartan improves heart rate variability and heart rate turbulence in heart failure due to ischaemic cardiomyopathy. Journal of heart failure. $13(10): 812-7$.

15. Tuininga YS. Harry JGM, Crijns HJGM, Brouwer J, Berg MPV, Veld AJMV, Mulder G, Lie KI.(1995) Evaluation of importance of central effects of atenolol and metoprolol measured by heart rate variability during mental performance tasks, physical exercise, and daily life in postinfarct patients. Circulation. 92: 3415-3423.

16. Carpia A, F Antonomic, A Ciamei, V Roncoroni, D Sabino, L Fontana. (2000) Short-term effects of Losartan on autonomic nervous system. Am. J. Hypertension. 13(52): 149A.

17. Rabbia F, Silke B, Carra R, Milan A, Colle SD, Pugni C, Mulatero P, Chiandussi L. ( 2004) Heart rate variability and Baroreflex Sensitivity during fosinopril, Irbesartan and atenolol therapy in hypertension. Clin Drug Invest. 24(11).

18. Sevre K, Johan D, Lefrandt, Nordby G, Os I, Mulder M, Reinold OB Gans, Rostrup M, Smit JA.(2001) Autonomic Function in Hypertensive and Normotensive subjects. Importance of gender Hypertension. 37: 1351-1356.

19. Srinivasa J, Bhat MR, Adhikari P 2002. Comparative study of heart rate variability (HRV) during deep breathing in normotensive and hypertensive subjects. JАCM. 2002: 3(3): 266-70.
20. Prakash ES, Madanmohon T, Sethuraman KR, et al. (2005) Cardiovascular autonomic regulation in subjects with normal blood pressure, high- normal blood pressure and recent onset hypertension. Clin Exp Pharmacol Physiol. 32 : 488-494.

21. Pavithran P, Madanmohon T, Mithun R, Jomal M, Nandeesha H. (2008) Heart rate variability in middle aged men with new onset hypertension. Annals of Noninvasive Electrocardiol. 13(3) : 242-248.

22. Pancera P, Presciuttni B, Sansone S, Montagna L, Paluani F, Covi G, Lechi A. (1999) Effect of losartan on heart rate and blood pressure variability during tilt test and trinitroglycerine vasodilation. J hypertension. 17(4): 513-21.

23. Zaliunas R, Brazdzionyte J, Zabiela V, Jurkevicius R.( 2005) Effects of amlodipine and lacidipine on heart rate variability in hypertensive patients with stable angina pectoris and isolated left ventricular diastolic dysfunction. International journal cardiology. $101: 347-353$

24. Pavithran P, Madanmohon T, Mithun R, Jomal M, Nandeesha H. (2008) Heart rate variability in middle aged men with new onset hypertension. Annals of Noninvasive Electrocardiol. 13( 3) : 242-248.

25. Chowdhury S, Townend JN. (2001) Nitric oxide and hypertension: not just an endothelium derived relaxing factor. J Hum Hypertension. 15: 219-227.

26. Chowdhury S, Vaile JC. Fletcher J, et al. (2000) Nitric oxide and cardiac autonomic control in human. Hypertension. 36: 264-269.

27. Fauster V, Walsh RA, Harrington RA. ( 2011) Hurst's The Heart. 13th ed. China: Mc Graw Hill Company. 